\title{
LETTER
}

\section{Serum antibody response in critically ill patients with COVID-19}

\author{
Alban Longchamp ${ }^{1,2,3} \mathbb{0}$, Justine Longchamp ${ }^{1,3}$, Antony Croxatto ${ }^{4}$, Gilbert Greub ${ }^{4}$, Bienvenido Sanchez ${ }^{1 *}$, \\ Julie Delaloye ${ }^{1 *}$ on behalf of the Study Group
}

C 2020 Springer-Verlag GmbH Germany, part of Springer Nature

Dear Editor,

Coronavirus disease 19 (COVID-19) is caused by the severe acute respiratory syndrome coronavirus 2 (SARSCoV-2), which has affected more than 7 million people. High mortality rates were reported among elderly, and those requiring mechanical ventilation in the intensive care unit (ICU) [1, 2]. Understanding virus kinetics, and host serological response to SARS-CoV-2 is crucial to guide treatment, vaccine design and epidemiological control [3]. Currently, the antibody response against SARS-CoV-2 in critically ill patients remains unknown.

We analyzed the antibody response in 28 critically Ill patients, with laboratory confirmed SARS-Cov-2 infection, admitted to Sion hospital ICU (Switzerland), between March 8th and April 4th, 2020. Only patients with serum samples available at two different time points were included. Experimental methods are described in the electronic supplementary material.

The characteristics of the cohort are summarized in Table S1-3. 27 (96\%) patients required mechanical ventilation, and $5(19 \%)$ patients died. The median (IQR) intervals from symptom onset to ICU, and hospital admission were 9 (7-12), and 6 (4-10) days, respectively (Table S1). 26 (93\%) patients had available serum sample within \pm 2 days of ICU admission. Of them, 15 (58\%)

\footnotetext{
*Correspondence: bienvenido.sanchez@hopitalvs.ch; Julie.Delaloye@ hopitalvs.ch

${ }^{1}$ Department of Intensive Care Medicine, Centre Hospitalier du Valais Romand de l'Hôpital du Valais, (Site de Sion) Avenue du Grand-Champsec 80, 1951 Sion, Switzerland

Full author information is available at the end of the article
}

Alban Longchamp, Justine Longchamp, Bienvenido Sanchez and Julie Delaloye have contributed equally.

Members of study group are listed in acknowledgement section. already had virus-specific IgG antibodies. (Table S2, and Fig. 1a, b). The distribution of IgG seroconversion time from the date of ICU admission showed 2 peaks, the first one on admission, the second one about 20 days later. The median (IQR) time was 17 (1-22) days (Fig. 1b). The distribution of IgG seroconversion time, since the onset of symptoms showed only one peak, with the median (IQR) time at 10 (7-13) days (Fig. 1c). The proportion of patients with positive virus-specific IgG reached $96 \%$ over the follow-up period (Fig. 1a, and Table S2). At ICU admission, anti-N IgG levels correlated with the time from symptom onset (Fig. 1d). No association was seen between anti-N IgG levels and age, or any of the other clinical, and laboratory data assessed (Fig. S1). Interestingly, two patients had no, or weak IgG seroconversion in the ICU. One had leukemia, the other one lymphoma. They died on day 4, and 38 respectively. Patients were then split into survivors (that were discharged from the ICU), and non-survivors (that died in the ICU) in order to assess if IgG seroconversion correlates with survival. IgG levels tended to be higher in patients that remained alive (mean difference $\pm S D, 10.3 \pm 5.5$, Fig. 1e). This suggests that the antibody response correlates with virus neutralization, and functional protection [4]. Consistently, SARS-CoV-2 cycle threshold of viral RNA amplification was low during the first week of ICU stay, then gradually increased (Fig. 1f), simultaneously to the IgG seroconversion (Fig. 1g). Further large-scale studies documenting the antibody responses against different SARS$\mathrm{CoV}-2$ antigens (Protein N, protein S), and viral clearance are needed to confirm our findings.

In conclusion, similarly to mild infections [4], most patients with severe COVID-19 developed SARS-Cov-2 specific antibodies [5]. This data also suggest that the

\section{Springer}




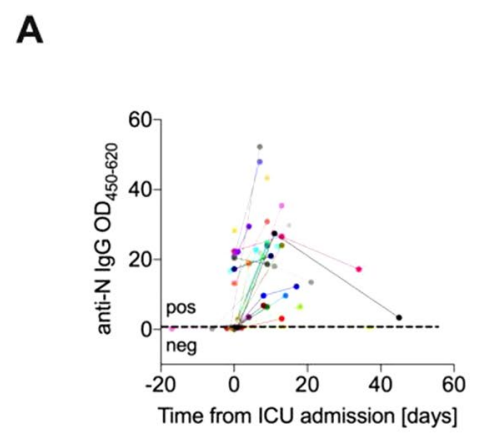

B

C

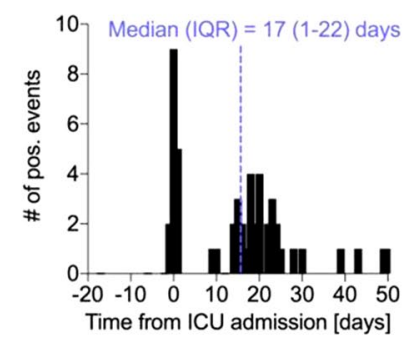

D

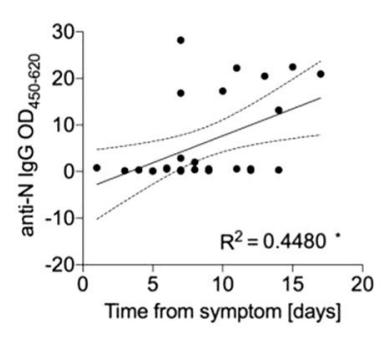

E

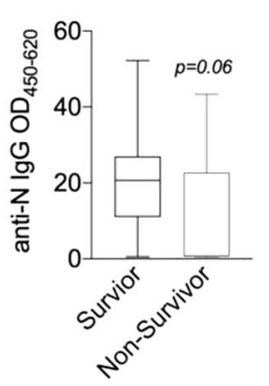

F

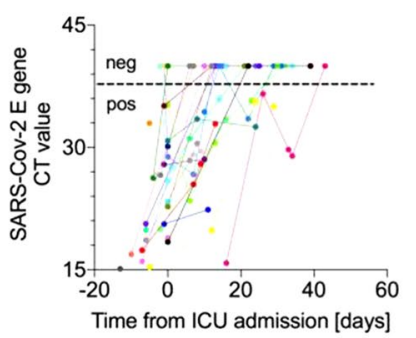

G

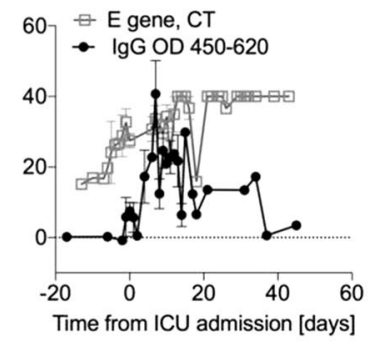

Fig. 1 Serological profile of critically ill patients with Covid-19. a anti-N IgG levels in patients over time since the admission to the ICU. b, c Interval from ICU admission (b), and symptom onset (c) in samples considered positive for SARS-CoV-2-specific IgG antibodies. d Unparametric Spearman's correlation between anti-N IgG levels at ICU admission, and corresponding time from symptom onset with their coefficient of determination $R^{2}$ and $p$ value. Dashed lines indicate $95 \%$ confidence intervals. (e) Pooled sera antibody levels in survivor versus non-survivors. $\mathbf{f}, \mathbf{g}$ Individual patient SARSCov-2 E gene threshold cycle (CT) values since admission to the ICU (f), and grouped with anti-N IgG profile $(\mathbf{g})$. Data are expressed as mean \pm SD. For negative $\mathrm{qPCR}, \mathrm{CT}$ values were arbitrary set at 40

severity of COVID-19 cannot be solely attributed to an impaired rate of seroconversion.

\section{Electronic supplementary material}

The online version of this article (https://doi.org/10.1007/s00134-020-06171-7) contains supplementary material, which is available to authorized users.

\section{Author details}

1 Department of Intensive Care Medicine, Centre Hospitalier du Valais Romand de l'Hôpital du Valais, (Site de Sion) Avenue du Grand-Champsec 80, 1951 Sion, Switzerland. ${ }^{2}$ Department of Biomedical Sciences, University of Lausanne, Lausanne, Switzerland. ${ }^{3}$ Department of Vascular Surgery, Centre Hospitalier Universitaire Vaudois and University of Lausanne, Lausanne, Switzerland. ${ }^{4}$ Department of Laboratory Medicine and Pathology, Institute of Microbiology, Centre Hospitalier Universitaire Vaudois, Lausanne, Switzerland. ${ }^{5}$ Service of Infectious Diseases, Institut Central Des Hôpitaux, Hôpital du Valais, Sion, Switzerland.

\section{Acknowledgment}

Dr. A. Longchamp reports receiving grant support from the Swiss National Science Foundation (SNSF PZOOP3-185927), and the Leenaards Foundation. Study Group: Livia Whiting, M.D., Séverin Jeanneret, M.D., Alix T. Coste, Ph.D., Alexis Dumoulin, Ph.D., Stéphane Emonet, M.D., Raymond Friolet, M.D.

\section{Author contributions}

$A L, J L, B S$, and JD designed the project. AC, GG, AD and SE performed the ELISA, and the GPCR. AL, JL, LW, AC, GG, AD, SE, and JD collected the data. AL,
$J \mathrm{~L}, \mathrm{AC}, \mathrm{BS}$, and JD analyzed the data. AL, JL, LW, SJ, GG, RF, BS, and JD wrote the manuscript.

\section{Compliance with ethical standards}

\section{Conflicts of interests}

All authors declare no conflicts of interest.

\section{Publisher's Note}

Springer Nature remains neutral with regard to jurisdictional claims in published maps and institutional affiliations.

Accepted: 30 June 2020

Published online: 8 July 2020

\section{Reference}

1. Yang X et al (2020) Clinical course and outcomes of critically ill patients with SARS-CoV-2 pneumonia in Wuhan, China: a single-centered, retrospective, observational study. Lancet Respir Med. 8(5):475-481. https:// doi.org/10.1016/S2213-2600(20)30079-5

2. Richardson S et al (2020) Presenting characteristics, comorbidities, and outcomes among 5700 patients hospitalized with COVID-19 in the New York City area. JAMA. 323(20):2052-2059. https://doi.org/10.1001/ jama.2020.6775 
3. Winter AK, Hegde ST (2020) The important role of serology for COVID-19 control. Lancet Infect Dis. 20(7):758-759. https://doi.org/10.1016/S1473 $-3099(20) 30322-4$

4. To KK et al. Temporal profiles of viral load in posterior oropharyngeal saliva samples and serum antibody responses during infection by SARS-CoV-2: an observational cohort study. Lancet Infect Dis. 2020;20(5):565-574. https://doi.org/10.1016/S1473-3099(20)30196-1

5. Zhao J, Yuan Q, Wang H et al (2020) Antibody responses to SARS-CoV-2 in patients of novel coronavirus disease 2019. Clin Infect Dis. https://doi. org/10.1093/cid/ciaa344 